\title{
Sexualbezogene Online-Fortbildung für Fachkräfte: Podcasts
}

\author{
Nicola Döringa
}

Zusammenfassung: Der vorliegende Praxisbeitrag stellt insgesamt zwanzig deutsch- und englischsprachige Podcasts vor, die sich mit sexualbezogenen Fragen befassen: fünf Sexpodcasts von und für den sexuellen Mainstream, fünf Sexpodcasts von und für sexuelle Minoritäten, fünf journalistische Sexpodcasts und fünf sexologische Sexpodcasts. Gemeinsamkeiten und Unterschiede dieser vier Gruppen sowie der besondere Informationswert von Sexpodcasts für Fachkräfte werden diskutiert.

Schlüsselwörter: Internet; Online-Fort- und Weiterbildung; Sexualberatung; Sexualkultur; sexuelle Gesundheit

\section{Sex-Related Online Training for Professionals: Podcasts}

Abstract: This practice-oriented article presents a total of twenty German and English language podcasts dealing with sexuality-related questions: five sexpodcasts by and for the sexual mainstream, five sexpodcasts by and for sexual minorities, five journalistic sexpodcasts and five sexological sexpodcasts. Similarities and differences between these four groups as well as the special information value of sexpodcasts for professionals are discussed.

Keywords: internet; online professional training; sexual counseling; sexual culture; sexual health

$\mathrm{Zu}$ den zahlreichen Online-Ressourcen, die für informelle sexualbezogene Fortbildung von Fachkräften zur Verfügung stehen (Döring 2018a), gehören Webvideos auf YouTube (Döring 2017a, 2017b, 2018b) sowie die noch stärker didaktisch aufbereiteten Webinare und verwandte E-Learning-Formate (Döring 2018c). Im Unterschied zu diesen audiovisuellen Formaten setzen

a Institut für Medien und Kommunikationswissenschaft, Technische Universität Ilmenau

Z Sexualforsch 2018; 31; 385-395

(c) Georg Thieme Verlag KG Stuttgart · New York

ISSN 0932-8114

DOI 10.1055/a-0760-6702 
die im vorliegenden Beitrag behandelten sexualbezogenen Audio-Podcasts ${ }^{1}$ rein auf das gesprochene Wort.

\section{Podcasts}

Bei einem Podcast handelt es sich um eine oft wöchentlich oder monatlich aktualisierte Serie von Audio-Dateien, die über das Internet bezogen und auch abonniert werden kann. Die Bezeichnung Podcast setzt sich als Kofferwort zusammen aus Bestandteilen von „iPod“ (bekannter MP3-Player, der Audio-Dateien abspielt) und „Broadcasting“ (Verbreiten einer Radiosendung). Heute werden Podcasts eher auf dem Smartphone gehört. Während Webvideos und Webinare auch visuelle Aufmerksamkeit verlangen, können Podcasts, die nur auditive Aufmerksamkeit erfordern, noch besser nebenbei genutzt werden. Nachteilig am Podcast-Format ist jedoch, dass man auf der linearen Tonspur nicht querlesen kann und somit für die typischerweise 30bis 90-minütigen Episoden entsprechend viel Zeit und Geduld mitbringen muss.

Der Begriff „Podcast“ wurde 2004 geprägt. Seitdem haben sich Podcasts immer stärker verbreitet. Sie werden von Radiosendern, aber auch privaten Internetnutzenden angeboten und behandeln ein breites Spektrum an Themen. Anleitungen zur Podcast-Produktion finden sich online und in Buchform (z.B. Hagedorn 2016). Es existieren diverse Online-Portale, auf denen man nach Podcasts suchen und diese auch abonnieren kann, allen voran der internationale iTunes-Store (https://www.apple.com/de/itunes/podcasts/), der nur mit der entsprechenden kostenlosen iTunes-Software nutzbar ist, sowie das deutschsprachige Podcasting-Portal Podcast.de (https://www. podcast.de/). Auch Online-Musikstreaming-Dienste wie Spotify (https:// www.spotify.com/de/), SoundCloud (https://soundcloud.com/) und Deezer (https://www.deezer.com/de/) und Online-Radio-Plattformen wie Stitcher (https://www.stitcher.com/) halten Podcasts bereit. Zudem werden Podcasts oft auf eigenen Websites veröffentlicht und können mit der Suchbegriffskombination „[Thema] Podcast“ über Suchmaschinen wie Google gefunden werden.

\section{Sexpodcasts}

Auf Podcast.de werden rund 50 und im iTunes-Store mehr als 100 Podcasts zum Thema Sex angeboten. Die meisten Sexpodcasts sind kostenlos nutzbar, denn sie werden durch Spenden, Werbung und Sponsoring finanziert und dienen den Podcaster_innen zudem zu Marketing-Zwecken (z.B. für ihre Bücher, Beratungsangebote oder Bühnenshows). Sexpodcasts gehören

\footnotetext{
${ }^{1}$ Neben den typischen Audio-Podcasts existieren auch Video-Podcasts.
} 
laut iTunes-Charts (http://www.itunescharts.net/ger/) regelmäßig zu den meistgehörten deutschsprachigen Podcasts.

Im Folgenden werden vier Gruppen von Sexpodcasts vorgestellt und in ihrer Bedeutung für die Fortbildung von Fachkräften diskutiert: Sexpodcasts von und für den sexuellen Mainstream, Sexpodcasts von und für sexuelle Minoritäten, journalistische Sexpodcasts und schließlich sexologische Sexpodcasts. Zu allen vier Gruppen werden exemplarisch jeweils fünf besonders populäre Angebote herausgegriffen. Berücksichtigt werden dabei nur deutsch- und englischsprachige Sexpodcasts, die aktuell regelmäßig neue Episoden veröffentlichen und von denen bereits mindestens 20 Episoden vorliegen.

\section{Sexpodcasts von und für den sexuellen Mainstream}

Zu den bekannten deutschsprachigen Podcasts, die primär aus der Perspektive von Privatpersonen aus dem sexuellen Mainstream (d.h. heterosexuell, cisgender) das Thema Sex behandeln, gehören „Beste Freundinnen“, „Besser als Sex“, „Oh, Baby! “ und „Sex Tapes“. Der reichweitenstärkste englischsprachige Sexpodcast dieser Gruppe heißt „Guys we $\mathrm{f}^{* * * *} \mathrm{~d}^{*}$.

\section{„Beste Freundinnen“}

In ihrem Sexpodcast „Beste Freundinnen“ (https://beste-freundinnen.podigee.io/) betrachten Max und Jakob (beides Pseudonyme) in über 90 Episoden das Sexual- und Liebesleben aus dezidiert männlicher, heterosexueller Perspektive: „Warum Mann trotz Beziehung Bock hat mit anderen Frauen zu schlafen“, „Warum Männer One-Night-Stands so toll finden“ oder „Wenn der Lachs schlaff im Netz hängt" lauten aktuelle Episodentitel. Es geht ganz häufig um vermeintlich typische Geschlechterunterschiede beim Flirten und beim „Bimsen“, was einerseits Identifikationspotenzial bietet („Das kennt jeder Mann“), andererseits oft klischeehaft wirkt. Die betonte Selbstironie („Beim Aufnehmen dieser Folge mussten wir mehrmals lüften, um unsere aufgeblasenen Egos rauszulassen“) mögen manche witzig, andere eher aufgesetzt finden.

Während Max mit festem Job, Freundin und Kind im Reihenhaus lebt, ist Jakob Single, karriereorientiert und hat gerade sein Psychologiestudium abgeschlossen. Beide sind schon lange gut befreundet und schreiben auch Bücher (Max und Jakob 2017, 2019). Sie haben den Anspruch, ehrliche Einblicke in „männliches Denken“ über Sexualität (einschließlich „Machogebärden“) zu bieten, über das man sich aufregen könne, das aber eben - nach ihrer Erfahrung - weit verbreitet sei.

\section{„Besser als Sex“}

Der Sexpodcast „Besser als Sex“ (https://podtail.com/de/podcast/das-neuesexvergnugen) - früherer Name: „Sexvergnügen“ (https://podtail.com/ 
de/podcast/sexvergnugen/) - bringt es seit 2016 auf insgesamt knapp 80 Episoden. Leila Lowfire (auch als Nacktmodell tätig) und Ines Anioli (früher Radiojournalistin, heute YouTuberin) unterhalten sich ganz offen über eigene sexuelle Erfahrungen sowie die ihrer Freundinnen und gehen auf Hörer_innenfragen ein. „Wie lange sollte Sex dauern?“, „Was ist so geil an Fisting?“, „Ist es okay die Ex meines Freundes zu bumsen?“ heißen einzelne Folgen. Dieser Sexpodcast widersetzt sich tradierten Geschlechtsrollenerwartungen an weibliche sexuelle Zurückhaltung mit bewusst vulgärem Sound, Spaß an Provokationen und sexuellen Protzereien und nicht zuletzt schamlosem Exhibitionismus. Auch wenn beide Podcasterinnen Bisexualität erkunden, liegt der Fokus meist auf Lust und Last mit dem Heterosex.

Der Podcast polarisiert das männliche Publikum: Die einen (z. B. Rocket Beans TV 2018) stören sich an seiner Geschmacklosigkeit, Niveaulosigkeit und der Objektifizierung von Männern, über deren mangelnde sexuelle Talente und defizitäre „Schwänze“ ausgiebig gelästert wird („Und dann schiebt dieser Italiener seine Spaghetti in mich rein, für so zehn, zwanzig Sekunden, und kommt“). Die anderen (z. B. Steinkuhl 2017) loben seine Ehrlichkeit, Tabulosigkeit und den hohen Unterhaltungswert. Auch aus weiblicher und feministischer Perspektive ist die Herangehensweise zwiespältig: Die auftrumpfende Art der beiden Podcasterinnen kann inspirierend, empowernd und befreiend wirken (etwa wenn die eigenen Genitalien als „große Schatzkiste“ gefeiert werden), aber auch effekthascherisch, anbiedernd und kommerziell („Wir sind zwei heiße Muschis, die kein Blatt vor den Mund nehmen, und wir reden übers Ficken“). Ebenso wie „Beste Freundinnen“ gehört „Besser als Sex“ laut iTunes-Charts (http://www.itunescharts.net/ ger/) aktuell zu den Top 30 der meistgehörten deutschsprachigen Podcasts.

\section{„Oh, Baby!“}

Der Sexpodcast „Oh, Baby!“ (http://www.ohbaby-podcast.de/) umfasst mehr als 50 Episoden, in denen laut Selbstbeschreibung „Isabel und ihre Freundinnen“ auf der Suche nach besserem Sex sind. Denn weder Isabel, die in glücklicher Beziehung mit Freund und gemeinsamem Kater am Münchner Stadtrand lebt, noch ihre Single-Freundinnen haben offenbar automatisch das tolle Sexleben, von dem sie träumen. Also nehmen sie das Publikum mit auf Streifzüge durch Bars, Dating-Apps und Cafés, sprechen mit Freund_innen, Fremden und Fachleuten wie etwa der Wiesbadener Frauenärztin Dr. Sheila de Liz. Der Podcast ist relativ aufwändig produziert und geschnitten.

Inhaltlich geht es um Fragen wie „Das Vorspiel - wichtig oder überbewertet?“, „Selbstbefriedigung: Wie wir es uns am liebsten machen“, „Unsere Sexfantasien - Wilde Orgien oder romantisch am Strand?“. Isabel und ihr Partner äußern sich selbst sehr persönlich. Zudem kommen Zuhörer_innen zu Wort, die Text- und Sprachnachrichten per WhatsApp oder über die Upspeak-App (https://www.upspeak.de/de/communities/ohbaby) einreichen. Auf diese Weise wird ein breites Spektrum an intimen Erfahrungen geteilt, seien es Erinnerungen an die ersten Selbstbefriedigungsversuche oder Details aus den eigenen Lieblingsmasturbationsfantasien. In der 
Episode „Sex, der dein Leben für immer verändert“ berichten Isabel und ihr Freund darüber, wie sie von ihrer aktuellen Schwangerschaft erfuhren. Ein Zuhörer schildert seinen ersten Sex mit einem Mann, bei dem ihm plötzlich sein Schwulsein bewusst wurde. Eine Zuhörerin erzählt von ungeschütztem Sex, in dessen Folge sie aufgrund einer zunächst unentdeckten ChlamydienInfektion unfruchtbar wurde.

Im Vergleich zu „Besser als Sex“ wirkt dieser Sexpodcast deutlich bodenständiger, manchmal gar etwas betulich und bieder. Wenn der Podcast beispielsweise Ausflüge in die Welt der Online-Fetische oder der OnlineSexarbeit unternimmt, dann fällt das für die Macherin ausdrücklich nicht unter das normale Spektrum sexueller Vielfalt, sondern es wird normierend dazugesagt, dass diese Themen „nichts für schwache Nerven“ seien, dass es hier um die „Abgründe des Internets“ und um das „krasseste ,Oh, Baby“Interview“ gehe.

\section{„Sex Tapes“}

Die lebenslustigen Berlinerinnen und guten Freundinnen Lili und Lotte (reale Vornamen) erkunden in ihrem Sexpodcast „Sex Tapes“ (https:// sextapes-podcast.de/) in bislang rund 20 Episoden diverse Aspekte des Sexuallebens, sei es Analsex mit Penetration des Mannes, Sex während der Menstruation oder feministische Pornografie. Die beiden Podcasterinnen sind unverkennbar akademisch gebildet. Guter Sex ist für sie „ResonanzSex“ im Sinne der Resonanztheorie des Soziologen Hartmut Rosa (Rosa 2016). Wenn sie vom ersten Besuch einer Sexparty berichten, dann scheint ihr Erleben in erster Linie nicht von Geilheit, sondern von ethnografischer Neugier geprägt. Lustvoll und sinnlich wird es indessen in der Episode „Was ist dein Muster im Bett?", in der sie sich detailliert ihre sexuellen Vorlieben erzählen und dabei das jeweils individuelle sexuelle Skript unter Lachen und Staunen schrittweise durchgehen. Ihre Sexpodcast-Philosophie erklären sie im Interview mit dem Podcast „Viertausendhertz“ (https://viertausendhertz.de/frq21/?utm_source=sextapes-podcast.de).

\section{Diskussion zu Sexpodcasts von und für den sexuellen Mainstream}

Die hier vorgestellten reichweitenstärksten deutschsprachigen Sexpodcasts bedienen insofern den Mainstream, als sie fast durchgängig von jungen, weißen, cisgender und heterosexuell identifizierten Erwachsenen betrieben werden. Es sind in der Regel mindestens zwei Personen am Mikrofon, die sich gegenseitig befragen, kommentieren und zuweilen widersprechen. Typisch ist auch, dass alle Podcaster_innen sehr persönliche Erfahrungen aus ihrem eigenen Leben teilen. Mit Ausnahme der Macherinnen von „Besser als Sex“, die als Nacktmodell und Journalistin bereits anderweitig in der Öffentlichkeit standen, bleiben alle anderen oben genannten Sexpodcaster_ innen aus Deutschland bewusst anonym.

Das englischsprachige Angebot an Sexpodcasts von und für den Mainstream ist um ein Vielfaches größer, weist aber eine ähnliche Machart auf. 
Der führende Sexpodcast aus den USA heißt „Guys we f****d“ (https:// soundcloud.com/guyswefucked) und stammt von Corinne Fisher und Krystyna Hutchinson. Die Podcasterinnen teilen in bislang 250 Episoden sexuelle Erfahrungen und plaudern mit Männern, mit denen sie Sex hatten. Sie kritisieren gesellschaftliche Verhältnisse rund um Sexismus, sexuelle Doppelmoral und sexuelle Gewalt. Da beide auch als Comedy-Duo auftreten, stellt der Podcast eine Mischform aus Erfahrungsbericht und Unterhaltungsshow dar. Viele ihrer Sexpartner, die ebenfalls aus der Entertainment-Branche stammen, sind am Mikro ganz unbefangen. Der Impetus des Sexpodcasts ist ausdrücklich feministisch, es geht um Anti Slut-Shaming. Die Podcasterinnen haben inzwischen auch ein Buch veröffentlicht (Fisher und Hutchinson 2017). Ihre Philosophie erklären sie im Interview mit dem YouTube-Kanal „BUILD“ (https://www.youtube.com/watch?v=aVa5rfx6U-o).

Alle genannten Sexpodcaster_innen, die sich primär als Privatpersonen mit ihren sexuellen Erfahrungen zeigen, kritisieren das mangelnde Sprechen über authentische sexuelle Erfahrungen und möchten dieses Schweigen brechen. Sie treffen damit teils auf Kritik oder sogar auf Hass. Insbesondere die Frauen, die sich öffentlich sexuell explizit äußern, erhalten regelmäßig Beschimpfungen und Gewaltdrohungen. Sie stoßen aber auch auf großes Interesse beim Publikum, das sich seinerseits nicht selten mit Erfahrungsberichten und sexuellem Beratungsbedarf an die Podcaster_innen wendet.

Für Fachkräfte im Bereich sexueller Gesundheit bietet diese Gruppe von Sexpodcasts reichhaltiges zeitgenössisches Material zu sexuellen Erfahrungen, Fragen und Ausdrucksweisen junger, überwiegend urbaner und akademisch gebildeter Mainstream-Erwachsener, darunter mehrheitlich Frauen. Diese sexuell aktive Zielgruppe schafft sich in Sozialen Medien offenbar im Zuge von Peer-to-Peer-Kommunikation über Sexpodcasts selbst Aufklärungsressourcen, da hier eine Versorgungslücke besteht: Die professionelle Sexualpädagogik, die über Schulen stattfindet, fokussiert traditionell auf das Jugendalter und erreicht Erwachsene kaum. Und im Unterschied zu den Infrastrukturen von und für sexuelle Minoritäten bestehen für den Mainstream weniger Anlaufstellen und Foren für dezidiert sexualbezogenen Austausch und sexuelle Bildung. In der praktischen Arbeit können Fachkräfte ausgewählte Sexpodcasts oder auch einzelne Episoden ihrer Klientel als Denk- und Gesprächsanstöße empfehlen. Zudem bietet sich durch die bereits stattfindende Zusammenarbeit von Fachleuten wie Ärzt_innen, Psycholog_innen oder Pädagog_innen mit Sexpodcast die Möglichkeit, Aufklärungsbotschaften an diese Zielgruppe zu richten.

\section{Sexpodcasts von und für sexuelle Minoritäten}

Wer Sexpodcasts von und für sexuelle Minoritäten und Szenen sucht, stößt vor allem im englischsprachigen Raum auf ein breit gefächertes Angebot, aus dem hier fünf Beispiele herausgegriffen seien:

- Im Sexpodcast „Polyamory Weekly“ (http://polyweekly.com/category/ podcast/) behandelt die Aktivistin Cunning Minx mit ihrem Modera- 
tionsteam seit 2005 Fragen rund um Polyamorie und konsensuelle NichtMonogamie. In den inzwischen mehr als 550 Folgen geht es beispielsweise um Hierarchien und Machtverhältnisse in Poly-Beziehungen, um den Umgang mit Eifersucht oder um Erfahrungen in der Swinger-Szene.

- „Proud to be Kinky“ (https://proudtobekinky.libsyn.com/podcast) ist ein Sexpodcast mit über 70 Folgen aus der BDSM-Szene. Er wird von einer kleinen Freundesgruppe betrieben, darunter das Pärchen Viv und Blue Ben sowie Bakji Ben und Floss_84 (Eleanor). Thematisiert werden unter anderem szenespezifische Fragen der Partner_innensuche, die Aushandlung von dominanten und submissiven sexuellen Rollen und von Konsens, Dos and Don'ts der Fetisch-Szene sowie Coming-out-Prozesse.

- Der Sexpodcast „Talk about Gay Sex“ (http://talkaboutgaysex.libsyn. com/) von Steve V. Rodriguez, Jeremy Ross Lopez und Steve Carpenter widmet sich in bislang rund 70 Folgen der schwulen Szene, etwa der Partnersuche über Dating-Apps, One-Night-Stands, HIV- und STI-Prävention, schwuler Pornografie und Sexarbeit. Episode 50 trägt den Titel „Why the f*ck won't he text me back?" und behandelt das Problem, dass manche Männer sich sogar nach dem gelungensten Date und heißesten Sex einfach nicht mehr zurückmelden und damit Ratlosigkeit und Frustration hinterlassen.

- Mit „QueerWOC“ (http://queerwoc.com/) schaffen Money und Nikeeta, die sich als schwarze queere Frauen vorstellen, einen Raum für den Austausch von queeren Women of Color (WOC), die ihre Fragen, Themenwünsche und Kommentare einreichen können. Der Podcast umfasst rund 50 Folgen und behandelt Dating-Dilemmata, Beziehungsprobleme, Sexpositionen und Sextoys. Öfter aber geht es um Fragen der queeren Identitätspolitik im Zusammenhang mit aktuellem und historischem Rassismus.

- Im „Queer Sex Ed Podcast“ (http://www.QueerSexEd.org/) wollen Sara und Jay Sexualaufklärung von und für die Queer-Community bieten. Dabei repräsentieren beide selbst Queerness auf vielen Ebenen gleichzeitig: Sie identifizieren sich als genderqueer (nämlich als femme nonbinary) und als trans*: Sara als Transfrau, Jay als trans-maskulin. Sie leben konsensuelle Nicht-Monogamie bzw. Polyamorie und praktizieren BDSM. Ihre Sexual- und Beziehungspartner_innen sind typischerweise ebenfalls queer identifiziert. Sara lebt zudem mit psychischen und physischen Behinderungen und stellt fest, dass weiße Hautfarbe die einzige Gruppenidentität ihrer Person konstituiert, die nicht mit Ausgrenzung, sondern mit Privilegien verbunden ist. In mehr als 30 jeweils ein- bis zweistündigen Episoden werden Fragen zu sexueller Gesundheit, Beziehungen, queeren Identitäten, BDSM und Polyamorie auf sehr hohem theoretischen und sprachlichen Niveau und mit politischem Anspruch durchdiskutiert. Es geht um Heteronormativität ebenso wie um Sextoys, um Trans-Dating und Sex-Positivität, um Untreue und ethische Kommunikation.

Für Fachkräfte besteht der Fortbildungswert dieser Angebote in dem Einblick in die Sichtweisen, Anliegen und Fragen der jeweiligen Szenen und 
Communitys - wobei natürlich Inhalte von Sexpodcasts aus dem angloamerikanischen Raum jeweils auf die kulturellen und rechtlichen Verhältnisse in Deutschland zu übertragen sind.

\section{Journalistische Sexpodcasts}

Medienhäuser haben sich dem Boom der Sexpodcasts angeschlossen. Vier deutschsprachige und ein englischsprachiges journalistisches Angebot seien vorgestellt:

- Der wöchentliche Sexpodcast „Eine Stunde Liebe“ von Deutschlandfunk Nova (https://www.deutschlandfunknova.de/podcasts/download/einestunde-liebe) bietet mehr als 330 Folgen. Hier werden unterschiedliche aktuelle wie zeitlose sexualbezogene Themen sehr frisch und ausgezeichnet recherchiert mit O-Tönen behandelt: Sexualassistenz für Menschen mit Behinderung, Therapie für sexuell übergriffige Jugendliche, Leben mit HIV heute, Pansexualität, Sexspielzeuge.

- Im wöchentlichen Sexpodcast „Ist das normal“ von ZEIT ONLINE (https:// www.zeit.de/serie/ist-das-normal) besprechen zwei Wissenschaftsredakteur_innen zusammen mit der Ärztin, Sexual- und Traumatherapeutin Dr. Melanie Büttner in bislang rund 50 Folgen Themen zwischen Sexualberatung („Muss man beim Oralsex verhüten?“) und Sexualpolitik („Der hohe Preis von käuflichem Sex“). Zu jedem Beitrag gibt es eine Liste von Quellen und wissenschaftlichen Studien. Der Sexpodcast, dessen Existenz vom „ZEIT“-Publikum in den Kommentaren teilweise als „Verschmuddelung“ kritisiert wird, ist um Legitimierung bemüht und erhebt den Anspruch „mit viel Wissen und absolut fundiert“ vorzugehen. Dabei bleibt er oft einer gefahren- statt ressourcenorientierten Sichtweise auf Sexualität verhaftet, orientiert sich nicht konsequent an dem in der Fachcommunity gängigen rechtebasierten Ansatz und reflektiert die eigene Position zu wenig.

- Der wöchentliche Sexpodcast „Im Namen der Hose“ vom Jugendkanal des Bayerischen Rundfunks PULS (https://www.br.de/mediathek/podcast/imnamen-der-hose-der-sexpodcast-von-puls/521) thematisiert in gut 20 Folgen eher praktische Themen wie Knutschen, Verhütung, Sex in Fernbeziehungen, Lecken und Fingern oder ein „spätes erstes Mal“, wobei die beiden Moderatorinnen O-Töne einholen und vielfach eigene Erfahrungen einfließen lassen.

- Im wöchentlichen Sexpodcast „Ladylike - Was Frauen wirklich wollen“ von 105‘5 Spreeradio (https://www.spreeradio.de/mediathek/podcasts/ ladylike-was-frauen-wirklich-wollen-id140856.html) plaudern Yvonne (lesbisch identifiziert und seit 11 Jahren in fester Beziehung) und Nicole (heterosexuell identifiziert, verheiratet und Mutter von zwei Kindern) seit rund 100 Folgen über ihre sexuellen Erfahrungen rund um Verlieben, Blasen, Abturner oder sexuelle Belästigung bzw. „\#MeToo“. Die Sendung ist als intimes Frauengespräch auf der Damentoilette inszeniert, eingeleitet von der Erkennungsmelodie der Kultserie „Sex and the City“. Das 
Ganze wirkt authentisch, einschließlich authentischer Wissenslücken, etwa wenn beide etwas naiv anmutend über „Geschlechtsumwandlungen" und deren Folgen spekulieren.

- „The Heart“ (https://www.theheartradio.org/) ist ein aus einem Montrealer Hochschul-Radiosender hervorgegangener preisgekrönter, von fünf Frauen betriebener Sexpodcast, der vor allem Emotionen und Intimität rund um Sexualität erkundet. Die Episoden sind aufwändig und mit künstlerischem Anspruch als Features kreiert - Hintergrundgeräusche, Küsse, Atmen, Flüstern und Pausen erzeugen eine dichte Atmosphäre. Herausragend ist beispielsweise die von Kaitlin Prest produzierte vierteilige Episodenreihe „No“ (https://www.theheartradio.org/no-episodes/), in der die Podcasterin eigene nicht-einvernehmliche sexuelle Interaktionen schmerzlich lebensnah wieder heraufbeschwört und dann ausführlich reflektiert. Nach zehn Jahren und 80 Folgen wurde „The Heart“ Ende 2017 eingestellt, wird hier aber aufgrund seiner Einzigartigkeit dennoch aufgeführt.

\section{Sexologische Sexpodcasts}

Die meisten Sexpodcasts dieser Gruppe sind englischsprachig. Sie stammen überwiegend von ausgebildeten Sexologinnen und wollen Einzelpersonen und Paaren Tipps zur Verbesserung ihres Liebes- und Sexuallebens geben.

- Dr. Emily Morse verfügt über einen Doktortitel in Human Sexuality und betreibt mit „Sex with Emily“ (http://sexwithemily.com/category/ podcast/) seit 2012 einen der langlebigsten und populärsten englischsprachigen Sexpodcasts mit über 430 Folgen. Mit ihren Gästen bespricht sie Dating und Sextoys, die Wirkung von Cannabis oder Schmerzen beim Sex und gibt Antworten auf Publikumsfragen. Die Podcasterin hat auch eine eigene TV-Show und inszeniert sich vor allem als Medienpersönlichkeit.

- Dr. Lindsey Doe ist als klinische Sexologin tätig und zugleich eine SocialMedia-Influencerin: Ihr YouTube-Kanal „Sexplanations“ gehört zu den führenden englischsprachigen Video-Aufklärungskanälen (Döring 2017a). Ihr gleichnamiger Audio-Podcast „Sexplanations“ (https:// soundcloud.com/user-863387665) bietet bereits mehr als 50 Folgen. Im Unterschied zu den relativ kurzen YouTube-Videos gehen die rund einstündigen Podcast-Episoden viel stärker ins Detail. Besonders interessant sind die selbstreferentiellen Folgen, in denen sie im Gespräch mit einem Kollegen (Episode 53), mit einer ehemaligen Patientin (Episode 39) und mit ihren Teenager-Töchtern (Episode 50 und 25) ihren Beruf als Sexologin reflektiert oder ihre Arbeit als YouTube-Aufklärerin kommentiert (Episode 41).

- Dr. Valeria Chuba, klinische Sexologin und Beziehungscoach, will mit ihrem Sexpodcast „Get Sex-Smart“ (http://podcast.askdrvaleria.com/ full-episode-list/) Männern, Frauen und Paaren Tipps für ein befriedigendes Sexualleben geben. Die rund 100 Episoden drehen sich um das 
Körperselbstbild, um sexuelle Erwartungen und Verhaltensweisen oder um sexuelle Passfähigkeit in der Beziehung.

- Esther Perel ist als klinische Psychologin tätig und u.a. auf Beziehungssexualität spezialisiert. In ihrem Podcast „Where should we begin?“ (https://www.estherperel.com/podcast) sind authentische Therapiesitzungen mit Paaren dokumentiert und kommentiert. In den bislang rund 20 Folgen geht es um die Aufarbeitung von Themen wie Fremdgehen, Lustlosigkeit, Impotenz und Unfruchtbarkeit.

- Tristan Taormino ist keine ausgebildetete Sexologin, sondern Sexualaufklärerin, Autorin und Filmemacherin. In ihrem Sexpodcast „Sex Out Loud“ (http://tristantaormino.com/sex-out-loud/about/) behandelt sie in knapp 300 Episoden die zeitgenössische sexuelle Kultur, umfasst dabei Mainstream und Spezialkulturen. Dazu führt sie Interviews mit namhaften Fachleuten aus Sexualtherapie und Sexualberatung, aus Wissenschaft, Kultur und diversen sexuellen Szenen, u. a. in der Episode vom 22. November 2013 mit Cunning Minx. Sehr interessant ist auch ihre Auseinandersetzung mit „\#MeToo“ und sexuellen Grenzverletzungen in sexpositiven Communitys.

\section{Fazit}

Die im vorliegenden Praxisbeitrag präsentierten 20 Sexpodcasts bringen es zusammen auf mehr als 2700 Episoden. Würde man diese nonstop durchhören, wäre man länger als ein Vierteljahr beschäftigt. Die Sexpodcasts sind somit erfolgreich in ihrem Anliegen, das Schweigen über sexuelles Erleben $\mathrm{zu}$ brechen. Ihre Inhalte sind vielfältig und besonders dann überzeugend, wenn authentische sexuelle Erfahrungen detailliert und reflektiert besprochen werden, wenn sie neue Sach- und Fachinformationen über unsere zeitgenössische sexuelle Kultur vermitteln und dabei auch Tipps zu weiterführenden Quellen bieten. Bemerkenswert ist, dass die Sexpodcast-Szene stark weiblich geprägt ist und oft feministische Perspektiven einbringt. Von den 20 vorgestellten Sexpodcasts werden 14 allein von Frauen betrieben, an vier wirken Frauen mit und nur zwei stammen allein von Männern. Generell wird beobachtet, dass Frauen verstärkt das Podcast-Format nutzen, um persönliche Geschichten - auch jenseits von Sexualität - öffentlich zu machen (McHugh 2017).

Insgesamt ist das Feld der Sexpodcasts unübersichtlich und boomend. Es braucht etwas Zeit, sich zu orientieren und individuell passende Angebote auszuwählen. Neben den inhaltlichen Schwerpunkten und der technischen Tonqualität mögen auch die Klangfarbe der Stimme und das sexuelle Vokabular der Podcaster_innen wichtige Auswahlkriterien sein. Denn durch die relativ lange Spieldauer und die Nutzung über Kopfhörer vermitteln Podcasts ein recht intimes Hörerlebnis. Ein Podcast-Abo kann dazu animieren, sich regelmäßig mit bestimmten Themen zu befassen und somit weiterzubilden. 
Die Nutzung von Podcasts mag zudem dazu anregen, ein eigenes Sexpodcast-Projekt zu starten. Wie bei allen Social-Media-Projekten kommt es aber auf ein tragfähiges, langfristiges Konzept für wöchentliche Folgen und eine ausreichend gute technische und organisatorische Umsetzung einschließlich Betreuung von Publikumsanfragen an (Hagedorn 2016). Sehr viele Sexpodcasts, auf die man bei Recherchen stößt, sind inzwischen längst nicht mehr aktiv. Umso anerkennenswerter sind Projekte, die seit Jahren laufen. So beantwortet der Sex-Kolumnist und Aktivist Dan Savage seit 2006 wöchentlich sexualbezogene Publikumsfragen in seinem Podcast „Savage Lovecast“ (https://www.savagelovecast.com/). Sexualwissenschaftliche Studien zu Sexpodcasts, ihren Inhalten, Betreiber_innen, Hörer_innen und Wirkungen stehen bislang aus.

\section{Literatur}

Döring N. Online-Sexualaufklärung auf YouTube: Bestandsaufnahme und Handlungsempfehlungen für die Sexualpädagogik. Z Sexualforsch 2017a; 30: 349-367

Döring N. Sexualaufklärung im Internet: Von Dr. Sommer zu Dr. Google. Bundesgesundheitsblatt Gesundheitsforschung Gesundheitsschutz 2017b; 60: 1016-1026

Döring N. Sexualbezogene Online-Fortbildung für Fachkräfte: Eine Einführung. Z Sexualforsch 2018a; 31: 97-100

Döring N. Sexualbezogene Online-Fortbildung für Fachkräfte: Webvideos. Z Sexualforsch 2018b; 31: $97-100$

Döring N. Sexualbezogene Online-Fortbildung für Fachkräfte: Webinare und andere E-LearningKurse. Z Sexualforsch 2018c; 31: 295-304

Fisher C, Hutchinson K. F*cked. Being Sexually Explorative and Self-Confident in a World That's Screwed. New York, NY: HarperCollins 2017

Hagedorn B. Podcasting. Konzept | Produktion | Vermarktung. Frechen: mitp 2016

Max \& Jakob. Beste Freundinnen. Wenn Männer über Frauen, Sex und den Sinn des Lebens reden. Köln: Bastei Lübbe 2017

Max \& Jakob. Kann ich nicht sagen, muss ich nackt sehen. Was Männer über Beziehung, Sex und Liebe denken. Köln: Bastei Lübbe 2019

McHugh S. Editorial: How Women Are Reclaiming Their Power in the Podcast Sphere. RadioDocReview 2017; 3(2) [Als Online-Dokument: http://ro.uow.edu.au/cgi/viewcontent.cgi?article=$1070 \&$ context $=\mathrm{rdr}]$

Rocket Beans TV. Sex-Podcasts, One-Night-Stands und Verhütungs-Tipps. Almost Daily \#336 mit Eddy, Florentin und Anja. YouTube 05.05.2018 [Als Online-Dokument: https://www.youtube. $\mathrm{com} /$ watch?v=7Vnbmob-05s]

Rosa H. Resonanz: Eine Soziologie der Weltbeziehung. Frankfurt/M.: Suhrkamp 2016

Steinkuhl H. Podcast „Sexvergnügen“: peinlich, eklig, affektiert - und ziemlich gutes Entertainment. Meedia 17.03.2017 [Als Online-Dokument: https://meedia.de/2017/03/17/podcast-sexvergnuegen-peinlich-eklig-affektiert-und-ziemlich-gutes-entertainment/]

Korrespondenzadresse

Prof. Dr. phil. Nicola Döring

Technische Universität Ilmenau

Institut für Medien und Kommunikationswissenschaft

Ehrenbergstr. 29 (EAZ 2217)

98693 Ilmenau

Nicola.Doering@tu-ilmenau.de 